\title{
BMJ Open Does the behavioural type-specific approach for type 2 diabetes promote changes in lifestyle? Protocol of a cluster randomised trial in Japan
}

\author{
Misa Adachi, ${ }^{1,2}$ Kazue Yamaoka, ${ }^{2}$ Mariko Watanabe, ${ }^{3,4}$ Asuka Nemoto, ${ }^{2}$ \\ Toshiro Tango ${ }^{2,5}$
}

To cite: Adachi M, Yamaoka K, Watanabe $\mathrm{M}$, et al. Does the behavioural type-specific approach for type 2 diabetes promote changes in lifestyle? Protocol of a cluster randomised trial in Japan. BMJ Open 2017;7:e017838. doi:10.1136/ bmjopen-2017-017838

- Prepublication history and additional material for this paper are available online. To view please visit the journal (http:// dx.doi.org/10.1136/bmjopen2016-014238).

Received 19 May 2017 Revised 5 September 2017 Accepted 27 September 2017

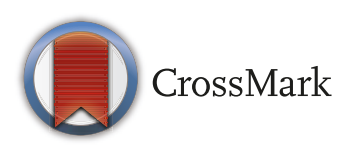

${ }^{1}$ Nutrition Support Network LLC, Kanagawa, Japan

${ }^{2}$ Teikyo University Graduate

School of Public Health, Tokyo, Japan

${ }^{3}$ Showa Women's University, Tokyo, Japan

${ }^{4}$ Prefectural University of Kumamoto, Kumamoto, Japan ${ }^{5}$ Center for Medical Statistics, Tokyo, Japan

Correspondence to Professor Kazue Yamaoka; kazue@med.teikyo-u.ac.jp

\section{ABSTRACT}

Introduction Type 2 diabetes (T2D) is a significant problem, and lifestyle modifications including self-management are important. We have developed a structured individual-based lifestyle education (SILE) programme for T2D. With attention now being paid to techniques to change behaviour, we recently developed a behavioural type-specific SILE (BETSILE) programme. We aimed to evaluate the effectiveness of the BETSILE programme compared with the SILE programme for reducing glycated haemoglobin $(\mathrm{HbA} 1 \mathrm{c})$ in patients with T2D and special behavioural types by a cluster randomised controlled trial.

Methods and analysis This is a 6-month cluster randomised controlled trial with two intervention arms (BETSILE vs SILE) provided in a medical care setting by randomising registered dietitians for patients with $\mathrm{T} 2 \mathrm{D}$ aged 20-79 years. Patients' behavioural types were classified into four types (BT1 to BT4) using an assessment sheet. We will perform independent trials for BT1 and BT2. The primary endpoint is a change from the baseline $\mathrm{HbA1C}$ value at 6 months. Differences between the SILE and BETSILE groups will be primarily analysed following the intention-to-treat principle. Crude and multivariate adjusted effects will be examined after adjusting for covariates, using a general linear mixed-effects model for continuous variables and a logistic regression mixed-effects model for dichotomous variables. Sample sizes needed were calculated assuming effect sizes of 0.42 and 0.33 for BT1 and BT2, respectively, an intraclass correlation of 0.02 , a significance level of $5 \%$ (two-sided), a power of $80 \%$, and equal allocation of clusters to the two arms, with each cluster having three BT1 patients for the SILE and BETSILE arms and six BT2 patients for the SILE and BETSILE arms. We will need 16 dietitians for each arm, and a total 288 patients will be required.

Ethics and dissemination This study has been approved by the Medical Ethical Committee of Teikyo University (No.15-222). Findings will be disseminated widely through peer-reviewed publications, etc.

Trial registration number UMIN 000023087; Pre-results.

\section{INTRODUCTION}

The prevalence of non-communicable diseases is increasing worldwide. Type 2 diabetes (T2D) is now considered to be a
Strengths and limitations of this study

- This is the first cluster randomised study to evaluate a behavioural type-specific approach to lifestyle education over a 6-month treatment period in Japan.

- The BETSILE approach was created by fusing the empirical knowledge of 10 or more registered dietitians in addition to the cognitive process in psychology (dual process theory).

- Outcome measures are clearly set using reliable clinical data and the test is adequately powered to detect effects on the outcomes.

- Blinding is not possible because of the type of intervention, which may affect the results.

- The study will be conducted in Japanese individuals only with two out of four behavioural types examined; therefore, generalisability will be somewhat limited.

global health issue, and preventing the onset and progression of diabetes is regarded as an important health measure in Japan. ${ }^{12}$ The ability to continuously self-manage lifestyle habits such as dietary habits and physical activities has a significant impact on the prevention of progression of T2D. The effectiveness of diabetes education, such as on diet and exercise, has been observed internationally, ${ }^{3-5}$ and standardised diabetes education programmes have been published. ${ }^{67}$ In recent years, it has been expected that health guidance will be applied to prevent the progression of diseases, including T2D, in Japan. There has been a gradual increase in the demand to motivate and promote effective behavioural changes in individuals by providing support for improvements in lifestyle issues, but little has been done to develop a standardised diabetes education programme. $^{8}$

Despite a proliferation of programmes and interventions to encourage and support dietary changes, bringing about lifestyle 
changes remains elusive. There are many barriers to lifestyle change. ${ }^{9}$ Psychotherapy, such as cognitive behavioural therapy and psychodynamic therapy, has been evaluated for its effectiveness in glycaemic control ${ }^{10}$; however, the effectiveness of behaviour type-specific interventions in achieving glycaemic control has not been examined and we expect that it may be effective in achieving such control.

The dual process theory (DPT) ${ }^{11}$ is widely known in the fields of cognitive and social psychology. Specifically, in the DPT the processes of 'System 1' are unconscious, rapid, automatic, and high capacity, while those of 'System 2' are conscious and slow. Human decision-making and behaviour are considered to be carried out by either System 1 or System 2. Daniel Kahneman explained that System 2 prevents the impulses of System 1 (self-control) and prevents various cognitive and judgement errors (cognitive bias) by easily entering into System 1; thus the two systems are mutually related. We can recognise differences among individuals in relation to these two systems-some people are more like System 2, while others are closer to System $1 .{ }^{12}$

Education directed toward improvement in lifestyles, such as dietary habits and physical activity, is known to be effective in preventing the onset and progression of diabetes. On the other hand, education incorporating the behavioural science approach has been identified as important to encouraging a behavioural change in patients, ${ }^{13}$ but the effect of practical education on lifestyles has been rarely examined. Both within and outside of Japan, few studies have examined improvements in lifestyle taking into account the study participants' characteristics. ${ }^{14}$

From our experience, lifestyle behaviour is associated with the systems described above and lifestyles can be changed depending on the types of behaviour according to those systems, especially for behaviour type 1 (BT1) and BT2. To use the mechanisms of DPT and to combine a behavioural type-specific approach, our experiences as registered dietitians may help to shift patients to more effective health choices. Therefore, we considered that a scientifically-based programme to evaluate effectiveness was essential. We previously developed a structured individual-based lifestyle education (SILE) programme for glycaemic control. ${ }^{1516}$ SILE utilises the food frequency questionnaire (FFQW82) to estimate nutritional intake for patients with non-communicable diseases. ${ }^{17}$ We examined the validity of SILE by a randomisation trial and confirmed its effectiveness. ${ }^{15-18}$

In order to improve the effectiveness of our educational programme, we propose a new behavioural type-specific SILE programme (BETSILE) corresponding to patients' behavioural types. ${ }^{19}$ Behaviour is classified into four types (BT1 to BT4) based on an assessment sheet of the 'Behavioural type specific chart'.

In our experiences as registered dietitians, in patients with BT1 and BT2 better behaviour could be achieved by the type-specific approach. As for patients with BT3 and BT4, when considering feasibility and benefit to patients, we decided to conduct trials using BT3 and BT4 in the future after determining the value of the BETSILE programme.

The aim of this study is to evaluate the effectiveness of the BETSILE with that of the SILE programme for T2D patients with BT1 and BT2 in reducing the level of glycated haemoglobin (HbAlc) by a cluster randomised controlled trial (RCT). If successful, the BETSILE programme can contribute to further improvement in preventing the onset and progression of T2D.

\section{METHODS AND ANALYSIS}

\section{Study design and setting}

The study is an open-label, multicentre, parallel-group comparison trial based on cluster randomisation (trial registration code: UMIN 000023087). An open-label design was necessary as the healthcare workers who performed the intervention at hospitals and clinics (hereafter referred to as 'medical settings') could not be blinded.

Patients enrolled in the trials for BT1 and BT2 will be classified using the 'Behavioural type-specific chart' (figure 1), and the two trials will be performed simultaneously in parallel as separate trials. Classification using this chart was done by four questions. Those four questions were selected from 14 assessment questions based on the result of pattern analysis using correspondence analysis (or Hayashi's Quantification Method 3) on awareness, motivation and behaviour of individuals who received outpatient dietary consultations. The results of the pattern analysis is shown in online supplementary figure 1.

With the collaboration of those in the medical setting, patients will be enrolled in the study. The registered dietitians who are employed in the medical setting will be allocated to each group of patients using a cluster randomised allocation. In each cluster, the registered dietitian works for both trials (for BT1 and BT2) and enrols three patients for BT1 and six patients for BT2 within the recruitment period. Each unit of the cluster (one registered dietitian/unit) comprises nine patients ( $\mathrm{n}=3$ for BT1 patients, $\mathrm{n}=6$ for BT2 patients). Cluster allocation will be used to avoid the possibility of contamination of information exchange within the same medical settings when different intervention methods are used. ${ }^{20}$

The intervention period is 6 months from randomisation (baseline). After the end of the intervention period, the participants will be monitored for an additional follow-up period of 6 months. Following the guidelines of Standard Protocol Items: Recommendations for Interventional Trials (SPIRIT), ${ }^{21}$ figure 2 shows a flow diagram of progression of the clusters and individuals through phases of the randomised trial. Registered dietitians will be randomised to either the BETSILE arm or the SILE arm. The primary endpoint is a change from the baseline value of HbA1c at 6 months. 


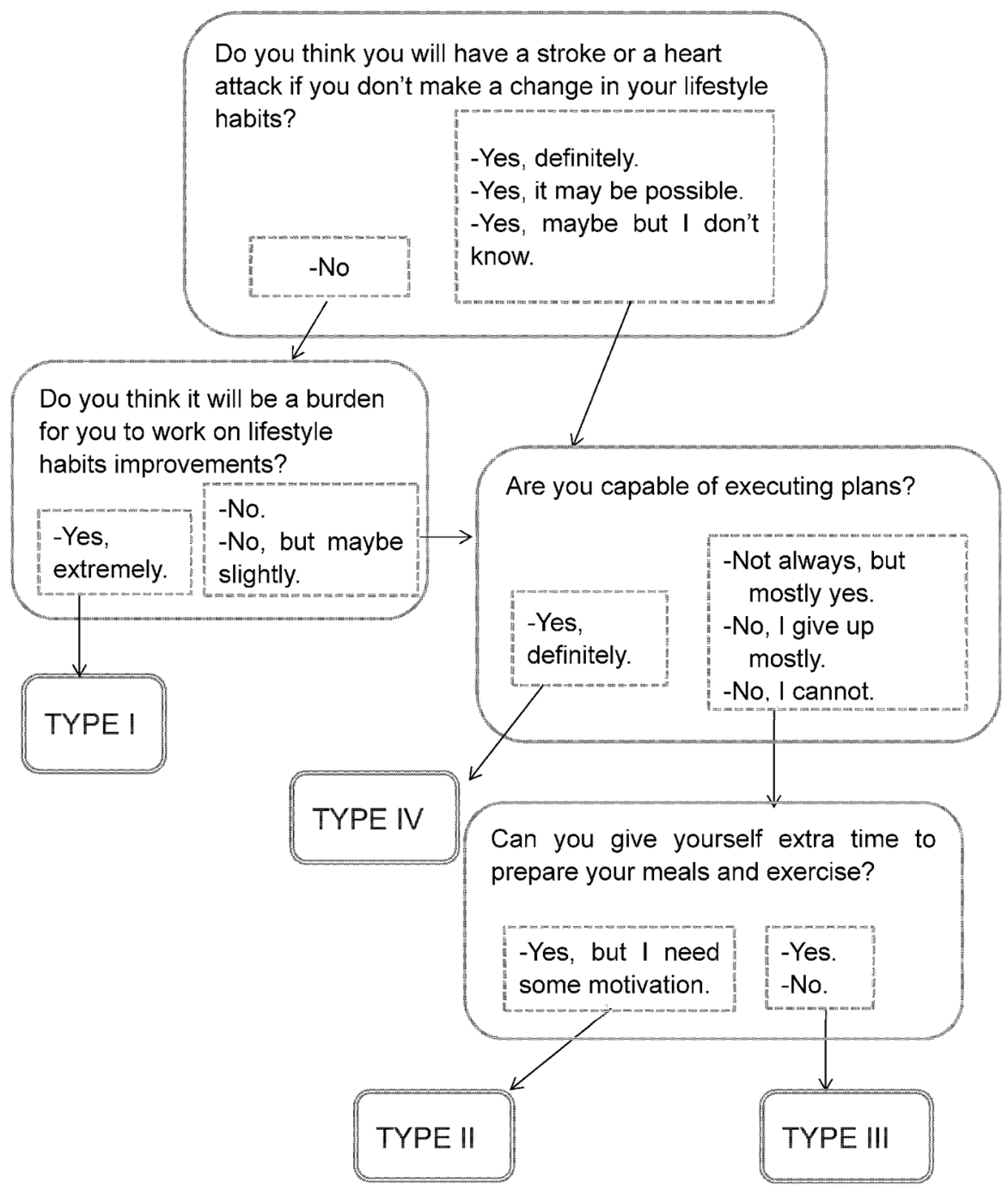

Figure 1 Behavioural type-specific chart.

Clinical data including that on each patient's HbA1c value will be obtained from the patient's primary physician. Lifestyle education by face-to-face and/or telephone sessions will be conducted several times during the study period by a registered dietitian. Outcome measures will be collected at baseline and at 3,6, and 12 months by the registered dietitian. (The measures at 3 and 12 months will be made if possible.) Specifically, behavioural changes in diet and exercise will be measured by the registered dietitian using the assessment sheet during the intervention period.

The trial was designed so that it can be reported according to the SPIRIT 2013 statement. The study will be carried out across multiple prefectures in the entire country of Japan. The Medical Ethical Committee of Teikyo University in Japan approved this study in 2016 (NO. Ethics Committee of Teikyo 15-222).

\section{Participants}

Initially according to the inclusion and exclusion criteria shown in Box 1, patients will be identified through examination of blood test results and medical screening by each patient's attending physician, nurse, or registered dietitian. Thereafter the patient's attending physician (or nurse or registered dietitian) will identify the patient's behavioural type. If eligible (BT1 and BT2), before any additional study procedures the participants will be requested to provide informed oral and written consent. Participants are 20 to 79 years old and receive care at the medical settings where they were diagnosed as having T2D according to an HbA1c value $\geq 6.5 \%$ (NGSP (formerly National Glycohemoglobin Standardization Program) criteria, referred to as NGSP below). The age of participants was selected based on consideration that among elderly people, particularly those over the age of 79 years, cognitive decline might be likely and affects issues of control of plasma glucose and the occurrence of severe hypoglycaemia.

\section{Recruitment and allocation}

Recruitment

We will recruit registered dietitians in several ways, including public invitation through the Dietitian Association of each prefecture, community websites for 
dietitians, and individual contact. The allocation officer at the study office allocates registered dietitians who have provided consent to participate sequentially based on the randomisation list created before the start of the study.

The registered dietitian (or primary physician, nurse) checks the inclusion and exclusion criteria of the patient, identifies the behaviour type using the behavioural type-specific chart, explains the contents of the study to the patient, and obtains consent if the patient is eligible. Patients who satisfy the selection criteria will be included in order without any selections.

\section{Assignment of participants}

Each registered dietitian will continue to recruit consecutive patients until the target number is reached.

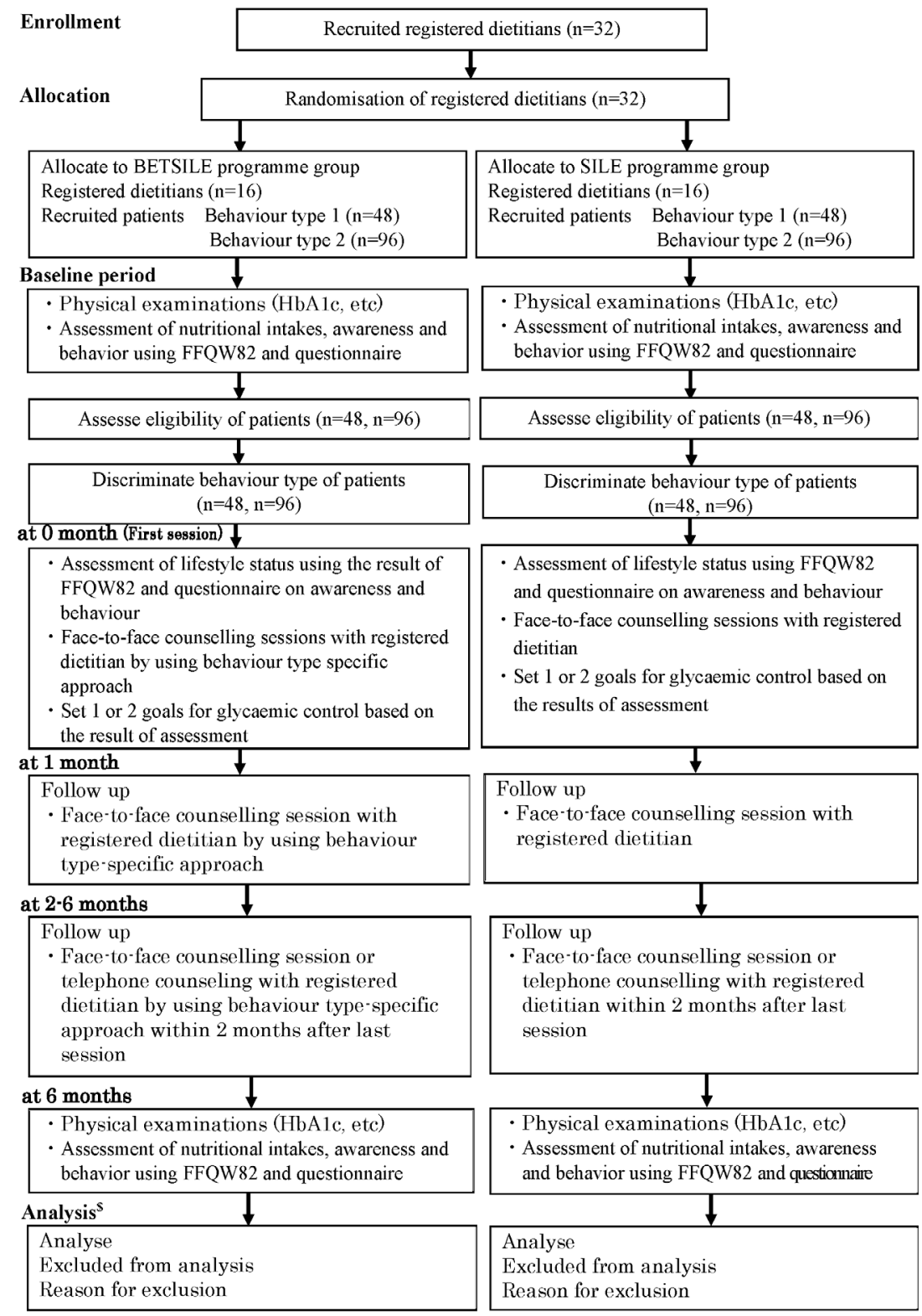

Figure 2. Flow diagram of progress of clusters and individuals through phases of randomized trial $\$$ : Until 12 months, 6 months monitoring period after the primary endpoint will be followed.

Figure 2 Flow diagram. BETSILE, behavioural type-specific structured individual-based lifestyle education; FFQW82, food frequency questionnaire; HbA1c , glycated haemoglobin; SILE, structured individual-based lifestyle education. 
Box 1 Eligibility criteria for participants in BT1 and BT2 trials. HbA1c, glycated haemoglobin.

Inclusion criteria
Type two diabetes (HbA1c $\geq 6.5 \%$ (NGSP))
Age 20 to 79 years
Receiving outpatient dietary consultation
Classified as BT1 or BT2
Not yet started therapy based on diabetes drugs Or recipient of oral
drugs or insulin injections for diabetes, and the attending physician
observed almost no change (difference in HbA1c <0.2\% in past
3 months) in HbA1c levels
Exclusion criteria
No consent obtained
Type 1 diabetes
Gestational diabetes
Diabetic retinopathy: proliferative retinopathy
Diabetic nephropathy: stage 3 or more
Determined by a doctor as having difficulty completing questionnaire

\section{Randomisation and blinding}

This trial is an open label trial. Cluster allocation is used as there is a possibility of information exchange in the same medical settings when different intervention methods are used. Cluster randomisation will be applied to avoid contamination bias. ${ }^{15}$ Collaborating registered dietitians will be randomised to the BETSILE programme or the SILE programme with the use of a randomisation list (random permutated blocks with block size 4) during October to December 2017.

\section{Interventions}

\section{BETSILE programme (intervention group)}

With this method, the behavioural type-specific approach is added to each counselling session with the contents and procedure of the SILE programme as a base (for details, see the SILE programme for the control group).

The method for the lifestyle intervention of the behavioural type-specific approach in BETSILE takes the DPT into account. Namely, lifestyle improvements do not rely solely on intra-individual factors such as one's own effort but rather extra-individual factors, such as environmental barriers.

In the DPT, System 1 is automatic, emotional, and sensory, and reactions cannot be controlled autonomously; erroneous recognition is also likely to occur. Conversely System 2 is related to logic, control, paying attention, thinking deeply, and endeavouring to achieve, but it is difficult to make this system work when the person participating feels busy or discouraged. In the DPT, decision-making and behaviour is dependent on these two systems. Some people's mode of action is similar to System 2 , while that of others is close to System $1 .^{12}$

As for the approach to improvements in lifestyle by the two behavioural types (BT1, BT2) in BETSILE, BT1 tends to be strongly influenced by factors that often hinder the appearance of System 2, while BT2 tends to be biased towards System 1. Considering these features, we adopted the following educational approach.

For those with BT1 it is easy to erroneously assume that 'complications do not occur to oneself'. Therefore, at the first interview, we begin by giving the patient correct information based on clinical data (plasma glucose levels and HbA1c) and evidence of the patient's actual condition and health risk, and correcting wrong assumptions. Also a BT1 individual tends to feel a considerable burden regarding bringing about lifestyle improvements because of feelings of being overwhelmed by a busy daily life, work, etc. In response to these feelings, at the first session using the BETSILE approach, the dietitian states: "If you achieve even one effective behaviour, your weight and clinical data (plasma glucose levels and HbAlc) may improve". Furthermore, in setting an action target, enough time must be given to consider the barriers to its execution and finding what can be done despite having a busy schedule or other barriers to success. These small steps reduce the sense of burden imposed by attempting lifestyle improvements and encourage the adoption of System 2 which may lead to subjective control.

BT2 is not good at subjective decision-making or aggressive behaviour, for instance, "I want to carry this out if possible" and "I can do it if I try, maybe". BT2 individuals are easily attracted to solutions such as health foods, health equipment, opinions of family members, etc. Even though there is a willingness to improve, execution tends not to last long. Therefore, for BT2, it is effective to encourage lifestyle improvements that will not be affected by outside influences by encouraging the use of System 2 by repeatedly paying attention to 'your health' at each session. Furthermore, the dietitian will recommend repeatedly that meals should be taken in accordance with the 'model menu', which shows suitable amounts for that individual, and to pay attention at meal time as to the appropriateness of the amount. Furthermore, dietitians will induce the participant to record dietary intakes. These practices may be related to System 2 (see table 1).

It is expected that these repetitions will eventually make the participant provide suitable information automatically using the function of System 1. It is effective to prepare environments so that good behaviour is natural in System 1 even if there has been no great effort or difficulty thinking for oneself. The content of the education is shown in supplementary table 1 .

\section{SILE programme (control group)}

We developed the SILE programme based on some of the strategies described in previous resources ${ }^{22-24}$ and updating the contents by new reference materials. ${ }^{25-27}$ Updated items were management of body weight, fat to energy ratio, and dietary fibre intake, which further strengthened the empowerment approach. The SILE programme was performed according to the nutrition care management (NCM) approaches with the additional educational approach for self-management and empowerment. NCM is a nutrition management system 
Table 1 Specific characteristics and approach to study participants according to type

\begin{tabular}{|c|c|c|}
\hline Type & Behavioural type 1 (BT1) & Behavioural type 2 (BT2) \\
\hline Characteristic of type & $\begin{array}{l}\text { BT1 does not recognise his/her illness and } \\
\text { seriousness of the health situation. } \\
\text { Eagerness for lifestyle change is low and } \\
\text { considers it as a burden. } \\
\text { Has tendency to hold on to beliefs. }\end{array}$ & $\begin{array}{l}\text { BT2 easily acts intuitively and emotionally. } \\
\text { Hope someone can improve his/her health } \\
\text { condition. } \\
\text { Mostly prioritises other people than considering his/ } \\
\text { herhealth. } \\
\text { BT2 tends to talk about assumptions such as 'I } \\
\text { would like to improve if possible', 'I can do it if I } \\
\text { want to do', etc. }\end{array}$ \\
\hline $\begin{array}{l}\text { Specific approach for } \\
\text { patients of each type }\end{array}$ & $\begin{array}{l}\text { Make sure to provide accurate information, } \\
\text { using blood test results and disease status. } \\
\text { Also have him/her think about what he/ } \\
\text { she wants to do to improve his/her health. } \\
\text { Mention health risks that could occur in } \\
\text { future that are realistic enough to occur in } \\
\text { his/her daily life. } \\
\text { Provide encouragement such as 'Achieve } \\
\text { continuation of at least one effective } \\
\text { behaviour to avoid failure in improving your } \\
\text { clinical data'. }\end{array}$ & $\begin{array}{l}\text { Provide encouragement such as 'Your health is } \\
\text { more important than taking care of others' and 'It is } \\
\text { your job to protect your own health'. } \\
\text { Show him/her suitable size of meals (per meal or } \\
\text { per day) with flip cards or food models. Advise } \\
\text { preparing meals with attention to the 'Model Menu'. } \\
\text { Encourage him/her to do 'self-reflection' as to } \\
\text { whether behaviour is bad or good for him/her. This } \\
\text { will enable him/her to continuously be aware of the } \\
\text { problem. } \\
\text { Repeatedly ask 'What is the goal you had wanted to } \\
\text { achieve?' }\end{array}$ \\
\hline
\end{tabular}

consisting of nutrition screening, nutrition assessment, and a nutrition care plan that involves implementation, monitoring, and evaluation. For the period from the first counselling session (start of intervention) up to 6 months ( \pm 1 month), the registered dietitian holds three or more counselling sessions. The second and third counselling sessions are held 1 month and 3 months ( \pm 1 month), respectively, after the first.

In the first session, assessment by the FFQW82 (see online supplementary table 2) is done to review daily dietary intakes. Based on the nutrition assessment the registered dietitian supports the participant to independently set goals for improvement of diet, physical activity, and glycaemic control. Physical activity will be measured by a questionnaire. The events that are likely to hinder the implementation of the primary goals are examined with the participant and solutions are discussed.

During subsequent sessions, the level of achievement of the short-term goal is reviewed by using the 'assessment and recording form' from the previous sessions. Results are applied in setting the next goals. The achievement of long-term goals is evaluated after 6 months. Behavioural changes in diet and exercise, which were set as goals at counselling sessions by dietitians, will be monitored at the consultation after 1 to 5 months as execution frequency. For this, the Behavior-Related Questionnaire (online supplementary table 3) was used.

\section{Training of health professionals}

We are preparing a SILE Programme Manual and a BETSILE Programme Manual. Each manual can be updated if additional specific procedures and ideas for assistance are developed. The registered dietitians undergo pre-briefing and training sessions using the study protocol and these manuals. They must sign a written pledge to comply with the study protocol. Moreover, registered dietitians must periodically refer to the contents of the study protocol to ensure that ethical and scientific nutrition education is provided. They are obligated to report promptly to the study office when a problem due to adverse events or management issues occurs.

The contents of the media shared by the SILE and BETSILE arms are prepared considering ethical and scientific nutrition education, and used during each counselling session.

\section{Study hypothesis}

The hypothesis underlying the study is that there is an improvement in glycaemic control when education is provided to two groups consisting of behavioural types 1 and 2 using the BETSILE programme compared with the SILE programme.

\section{Outcome measures}

Primary outcome

The primary outcome is the difference between the two groups in changes in HbAlc levels from baseline to $6 \pm 1$ months.

\section{Secondary outcomes}

Changes in body mass index, blood pressure, fasting plasma glucose, lipid profiles (low-density lipoprotein, high-density lipoprotein, triglycerides), and physical activity are assessed. As for physique, including height and weight, measurements will be made by a medical doctor or nurse and will be recorded, as will the baseline values 
from the clinical examination. Family medical history, current exercise, and smoking and drinking habits will be assessed using a self-rated questionnaire. Furthermore, changes in energy and nutrient intakes and the fat/ protein/carbohydrate energy ratio are assessed using the FFQW82. Moreover, the difference in the HbA1c levels between the two groups at the 12th month ( \pm 1 month) (at the completion of observation) from the first intervention compared with those at the time of study registration is examined as an exploratory analysis. The outcome assessments are blinded to statisticians.

\section{Patients' characteristics}

Patients' background characteristics (sex, age, complications, past medical history) are obtained from medical records and assessments.

As for the economic status of participants, in Japan questions on socioeconomic status in a daily clinical setting sometimes increases the rate of refusal to participate. If a patient feels economically distressed, a dietitian can provide practical advice to relieve the distress in most cases. However, even among those receiving welfare protection, few people are in extreme poverty.

\section{Statistical analysis}

Sample size

The registered dieticians are allocated to each group of participants using cluster randomised allocation. With $80 \%$ detection power and the two-sided significance level of $5 \%$, the hypothesis of the present study will be detected. The estimated improvement in the HbAlc levels for the BETSILE group compared with the SILE group was $0.5 \%$ or more for BT1 and $0.4 \%$ or more for BT2. These estimated HbAlc improvements were based on our dietitians' experience and a preliminary study (not published). Each registered dietitian has to assist three participants with BT1; therefore, in total 32 registered dietitians are required for the SILE (16) and BETSILE (16) groups. Similarly, each registered dietitian has to assist six participants with BT2; therefore 32 dietitians are required for SILE (16) and BETSILE (16). By amalgamating these, we need 32 registered dietitians for trials of BT1 and BT2 participants (288 patients in total). This study will be conducted in the usual clinical settings and the period is 6 months, which is not long. Therefore, the dropout rate will be expected to be low. Of those who do dropout, we will follow them clinically to obtain information on at least the primary outcome.

\section{Statistical analysis}

The primary target population for analysis consists of all cases (full analysis set, FAS) meeting the eligibility criteria described in the study protocol, which is carried out with the intention-to-treat (ITT) principle. Changes in HbAlc values from baseline to the time of evaluation (6 $6 \pm 1$ months) are compared between arms. Adjustment for confounding factors is examined using the generalised linear mixed-effects model. Confounding factors are age, physique, family medical history, current exercise, smoking and drinking habits, medication yes/no (diabetes therapy drug, hyperlipidaemia drug, and antihypertensive agents), and baseline values of evaluated items at the start of the study.

The binary variables of whether or not the diabetes control evaluation 'Target value from the perspective of preventing complications' is below HbAlc $7.0 \%$ (NGSP) ${ }^{23}$ will also be examined with the generalised linear mixed model (logistic regression). For the ITT analysis, the binary variable with the 'good' and 'bad' groups is used as the index of each continuous variable. For this, the Last Observation Carried Forward (LOCF) method, in which the value just before the available value is assigned, will be used. Multiple Imputation with the assumption of Missing at Random for the missing values as well as Ignorable Maximum Likelihood analysis will be conducted as sensitivity analyses. In addition, analysis using per protocol set will be also conducted.

All tests will be done with a significance level of $5 \%$ (two sided). All statistical analyses will be performed using SAS version 9.4 for Windows (SAS Institute, Inc, Cary, NC, USA).

\section{Time frame of the study}

Registered dietitians will be registered to participants in the study from October to December 2017, and all patients will be registered from October 2017 to December 2018. The baseline measurements will be started in October 2017. Data on the effect evaluation will be available in 2020. Intervention and final assessment at 6 months after inclusion will be performed for each patient sequentially.

\section{Data management}

Personal information obtained in this study will be coded and anonymised.

All the data and documents for the present study will be managed securely for 5 years from completion by the Nutrition Support Network LLC. All materials will be disposed of by shredding, and sufficient care will be taken to ensure that the names of individuals on the signed informed consent forms cannot be identified.

\section{Criteria for discontinuation of participation in the study or of the study}

1. If a participant withdraws consent for participation in the study.

2. If the attending physician determines that the continuation of the nutrition education is not preferable for an individual patient. At the moment, there is no reason that can be used to exclude a patient from the trial, which may minimise bias affecting the results of the study.

3 . If continuation of the study is considered difficult because of exacerbation of complications.

4. If the study protocol cannot be completely followed.

5. If a patient's physician determines that discontinuing the study is appropriate for the patient for other reasons. 


\section{Monitoring}

Information on when the study begins, the conduct of the study (sample size), ethical considerations, the occurrence of detrimental or adverse events, the results of the study, and registration of the study with a public database will be submitted to our ethics committee in an annual interim report. A report will also be submitted to the ethics committee on the conclusion of the study and when the final results are presented.

\section{Protocol amendments}

If any amendments are made, the Ethics Committee may be notified as necessary. The protocol has been updated as Vol. 2 in July 2017.

\section{Follow-up of adverse events}

This study involves implementation of a lifestyle programme to help persons with T2D to improve their lifestyle and to receive counselling from a registered dietitian. There is little likelihood of any health hazards. If any serious adverse events do occur, they will be reported in line with the standard operating procedure on Reporting Serious Adverse Events in Clinical Research.

\section{Ethics and dissemination}

The study is implemented in accordance with the Declaration of Helsinki and the ethical guidelines for medical research covering humans.

This study has been approved by the Medical Ethical Committee of Teikyo University (No.15-222).

The present study is an open-label study. All participants are given an ID and are anonymous. Thus, no information identifying specific patients is stored alongside study data. It is considered that harm to the patient will be rare because the examinations performed will be those undertaken in the usual medical examination. However, participants will be told that if they want to discontinue because of the psychological burden, they can do so by exercising their free will at any time. The required procedure for withdrawal will be followed. The same applies to collaborating registered dietitians.

\section{DISCUSSION}

Among most diabetic patients, it is essential for improvements in lifestyle to occur in order to maintain glycaemic control. Continuous and self-managed lifestyle change programmes in which effectiveness is evaluated exist in many other countries. However, there are few programmes other than SILE that have been evaluated for effectiveness by an RCT in Japan and that involves self-managed lifestyle change. Furthermore, the behavioural type classification method is the first of its kind to be used for operational classification based on analysed data on patients' intention to improve their health, patients' initiative in achieving goals, and application of patients' dietary knowledge. To our knowledge, there is little research in and outside of Japan on a lifestyle improvement programme that specifically considered participants' characteristics. Scientifically verified knowledge will encourage patients to change behaviours and in achieving such changes to reach even greater goals, and makes this research distinctive.

The advantage of this study is the addition of the new behavioural-type clustered approach to the SILE programme to prevent the serious effects of diabetes (BETSILE programme). The BETSILE programme can incorporate the behavioural science approach with information on characteristics of study participants and encourage behaviour modification, and is expected to facilitate more easily efforts toward glycaemic control by patients with diabetes in the future.

Contributors MA, KY, MW and TT were responsible for designing the research questions and drafting the study protocol. MA, KY and AN were responsible for data management. MA was responsible for management of dietary education. KY, TT and AN were responsible for statistical analyses. MA, KY and MW wrote the first draft of this paper. All authors read and approved the final manuscript.

Funding This study is financially supported by the Ministry of Education, Culture, Sports, Science and Technology in Japan Grant-in-Aid for Scientific Research Grant C in 2017 (Grant No. 17K00936). The work is also supported by the Health Science Centre Institute Foundation, Kanagawa, Japan. This funding source had no role in the design of this study and had no role during its execution, analyses, interpretation of the data, or in any decision to submit results.

Competing interests None declared.

Patient consent Obtained.

Ethics approval Teikyo University Review Board (Teikyo 15-222).

Provenance and peer review Not commissioned; externally peer reviewed. Data sharing statement The informed consent documents are available on request.

Open Access This is an Open Access article distributed in accordance with the Creative Commons Attribution Non Commercial (CC BY-NC 4.0) license, which permits others to distribute, remix, adapt, build upon this work non-commercially, and license their derivative works on different terms, provided the original work is properly cited and the use is non-commercial. See: http://creativecommons.org/ licenses/by-nc/4.0/

(c) Article author(s) (or their employer(s) unless otherwise stated in the text of the article) 2017. All rights reserved. No commercial use is permitted unless otherwise expressly granted.

\section{REFERENCES}

1. Ministry of Health, Labour and Welfare. A basic direction for comprehensive implementation of national health promotion. 2, 2012. http://www.mhlw.go.jp/file/06-Seisakujouhou-10900000Kenkoukyoku/0000047330.pdf. (accessed 15 April 2017).

2. Ministry of Health, Labour and Welfare. References on promotion of healthy Japan 21. 2nd ed, 2012:49-58. http://www.mhlw.go.jp/ bunya/kenkou/dl/kenkounippon21_02.pdf. (accessed 15 April 2017).

3. Norris SL, Lau J, Smith SJ, et al. Self-management education for adults with type 2 diabetes: a meta-analysis of the effect on glycemic control. Diabetes Care 2002;25:1159-71.

4. Yamaoka K, Tango T. Efficacy of lifestyle education to prevent type 2 diabetes: a meta-analysis of randomized controlled trials. Diabetes Care 2005;28:2780-6.

5. Chen L, Pei JH, Kuang J, et al. Effect of lifestyle intervention in patients with type 2 diabetes: a meta-analysis. Metabolism 2015;64:338-47.

6. Haas L, Maryniuk M, Beck J, et al. National standards for diabetes self-management education and support. Diabetes Care 2014;37 Suppl 1(Suppl. 1):S144-S153.

7. Department of Health England. National Service Framework for Diabetes: Standards. https://www.gov.uk/government/uploads/ system/uploads/attachment_data/file/198836/National_Service Framework_for_Diabetes.pdf. (accessed 25 Sep 2015). 
8. Nakano M, Moriyama M, Sakamaki H. A survey on the care and education system geared towards standardization of patient education for diabetes. Journal of Health Sciences Hiroshima University 2007:6:118-25.

9. Wilkinson A, Whitehead L, Ritchie L. Factors influencing the ability to self-manage diabetes for adults living with type 1 or 2 diabetes. Int $J$ Nurs Stud 2014:51:111-22.

10. Ismail K, Winkley K, Rabe-Hesketh S. Systematic review and meta-analysis of randomised controlled trials of psychological interventions to improve glycaemic control in patients with type 2 diabetes. Lancet 2004;363:1589-97.

11. Evans JS. Dual-processing accounts of reasoning, judgment, and social cognition. Annu Rev Psychol 2008;59:255-78.

12. Kahneman D. Thinking, Fast and Slow. New York, Farrar: Straus and Giroux, 2011

13. Pillay J, Armstrong MJ, Butalia S, et al. Behavioral programs for type 2 diabetes mellitus: a systematic review and network meta-analysis. Ann Intern Med 2015;163:848-60.

14. Saruwatari A, Kimura M, Nishi K, et al. An evaluation of a regional program for the prevention of diabetes mellitus considering the personality traits of a high-risk diabetic population. Japanese Jpn J Health Educ Promot 2013;21:26-36.

15. Adachi M, Yamaoka K, Watanabe M, et al. Effects of lifestyle education program for type 2 diabetes patients in clinics: study design of a cluster randomized trial. BMC Public Health 2010;10:742.

16. Adachi M, Yamaoka K, Watanabe M, et al. Effects of lifestyle education program for type 2 diabetes patients in clinics: a cluster randomized controlled trial. BMC Public Health 2013;13:467.

17. Adachi M, Watanabe M, Yamaoka K, et al. [Validity and reproducibility of a food frequency questionnaire with 82-food items (FFQW82) for nutrition education]. Nihon Koshu Eisei Zasshi 2010;57:475-85.

18. Yamaoka K, Adachi M, Watanabe M, et al. Practice and evaluation of lifestyle improvement - Towards the prevention of onset and progression of lifestyle-related diseases. (In Japanese) Tokyo: Asakura Syoten, 2015

19. Adachi M, Terasawa Y, Kaneko N, et al. Classification of patients in clinics for nutritional education of lifestyle change: trial of the evaluation for nutritional education with Behavior metric approach. Japan Journal of Behavior Metrics 2011;38:117-25.

20. Torgerson DJ. Contamination in trials: is cluster randomisation the answer? BMJ 2001;322:355-7.

21. Chan AW, Tetzlaff JM, Altman DG, et al. SPIRIT 2013 statement: defining standard protocol items for clinical trials. Ann Intern Med 2013;158:200-7.

22. Japan Diabetes Society. Evidence-based Practice Guideline for the Treatment for Diabetes in Japan. Tokyo: Nankodo, 2004.

23. American Dietetic Association. Medical nutrition therapy evidencebased guide for practice: nutrition practice guidelines for gestational diabetes. Chicago, IL: American Dietetic Association, 2001.

24. National diabetes education program: 4 steps to control your diabetes for life. https://www.cdc.gov/diabetes/ndep/pdfs/97ndep67-4steps-4c-508.pdf (accessed 25 Aug 2017)

25. Japan Diabetes Society. Evidence-based practice guideline for the treatment for diabetes in Japan. Tokyo: Nankodo, 2013.

26. Japan Diabetes Society. Evidence-based Practice Guideline for the Treatment for Diabetes in Japan. Tokyo Nankodo, 2016

27. American Diabetes Association. Standards of medical care in diabetes 2016. Diabetes Care 2016;39:30-42. 